\title{
Alternativas silvícolas para aumentar la rentabilidad de las plantaciones forestales
}

\author{
Silvicultural options to increase the profit of plantation forest \\ JORGE TORO VERGARA \\ Facultad de Ciencias Forestales, Universidad de Concepción, casilla 160-C, Concepción, Chile. \\ E-mail: jtoro@udec.cl
}

\begin{abstract}
SUMMARY
A site-specific approach to silviculture that seeks to increase the productivity and profitability of plantation forest is proposed in this paper. It is necessary to develop a silvicultural cycle that starts at the tree nursery and finishes after the final harvest. Gains in growth are possible at each step through the definition of a target plant for a specific site, together with the use of improved genetic material, all of which are crucial in developing this program. Soil and site data are an important consideration in the proposals for specific methods to improve the silvicultural regimes. These will avoid risks that could affect plantation growth and will be cost efficient. Responses that double the yield of plantations (Type II) have been obtained in contrasting soils located in the $8^{\text {th }}$ Region of Chile. It is consequently necessary to find the optimal factors to apply in each step of the silvicultural cycle in order to obtain equivalent results. Using site-specific silviculture, it will be possible to achieve success.
\end{abstract}

Key words: specific, silviculture, productivity, evaluation, sites.

\section{RESUMEN}

Se analiza la conveniencia de aplicar una silvicultura específica al sitio para aumentar la productividad de las plantaciones y la rentabilidad de ellas. Para esto, se requiere desarrollar un ciclo silvícola que se inicia en el vivero y finaliza después de la cosecha final. En cada etapa del ciclo se obtiene una ganancia en productividad. El empleo de una planta-tipo adaptada a las condiciones especiales de los sitios junto con un material genético de alta calidad, es la base para desarrollar esta nueva silvicultura.

El uso de la información del suelo y del sitio, es vital para preparar las prescripciones que facilitarán la implementación de regímenes silvícolas específicos. Esto permite evitar o disminuir los riesgos que pueden afectar a las plantaciones. Las respuestas del tipo II, que permiten duplicar el crecimiento, se han obtenido en suelos que tienen distintas capacidades productivas, por lo tanto, es necesario aplicar, en cada sitio, las combinaciones silvícolas más apropiadas en cada una de las etapas del ciclo silvícola propuesto. Estas acciones favorecerán el aumento de la productividad de las plantaciones y del sitio, como también un mejoramiento de la rentabilidad.

Palabras clave: silvicultura específica, productividad, evaluación, sitio.

\section{INTRODUCCION}

Desde hace más de una década, se desarrolla una silvicultura intensiva en Chile, con resultados variables, producto de los diferentes puntos de vista que tienen los profesionales forestales que pertenecen a distintas organizaciones y de la influencia que han ejercido sobre ellos los especialistas ex- tranjeros que han visitado el país, durante el período 1980-2002.

La fuente de inspiración para desarrollar una nueva silvicultura en Chile, basada en especies de crecimiento rápido, proviene de tres direcciones (1). La influencia inicial fue neozelandesa representada por Sutton y Fenton (1979), Will (1981), Summer (1983), Mead (1993), Mason y Carson, 
BOSQUE 25(2): 101-113, 2004

Alternativas silvícolas para aumentar la rentabilidad de las plantaciones forestales

(1998); luego los americanos, Gessel (1981, 1991, 1993 y 1995), Zobel (1982) y Allen (desde 1993) y últimamente los australianos, Turner y Lambert (entre 1992 y 2001), Nambiar y Carlyle (desde 1994). Ellos han dejado, con mayor o menor intensidad, huellas en el pensamiento científico de muchos profesionales nacionales y han contribuido a desarrollar un sector forestal nacional pujante, moderno y reconocido en el ámbito internacional.

Cada especialista extranjero ha aportado sus ideas desde áreas específicas: viveros, genética, economía, nutrición, suelos, sitios, silvicultura, ecofisiología, manejo de plantaciones, etc.

El enfoque que ha recibido el tema de productividad de sitios por parte de los especialistas chilenos es muy variado y el énfasis que han puesto en determinados aspectos se basa, principalmente, en el tipo de formación profesional que han recibido y en el especialista extranjero con que han interactuado (2-11).

El objetivo de este trabajo es analizar y proponer fórmulas biológicas que permiten hacer más rentables las plantaciones forestales, aplicando una silvicultura específica al sitio, con el propósito de aumentar la productividad no sólo de las plantaciones sino que también la del sitio. Esta silvicultura se diferencia de la intensiva, en que es sustentable bajo tres aspectos importantes: ecológico, económico y social. Para ello, se propone desarrollar un ciclo silvicultural, parte del cual las empresas nacionales están realizando, con los matices propios de cada una de ellas.

Se fundamenta esta propuesta en los resultados entregados por diversas investigaciones nacionales.

Antecedentes generales. Previo al análisis de los métodos que pueden aplicarse para aumentar la rentabilidad de las plantaciones, es necesario hacer una distinción entre la "silvicultura intensiva" (SI) que actualmente se emplea en nuestro país y una "silvicultura específica al sitio" (SES), que se convertiría en el elemento clave para elevar la productividad de las plantaciones y del sitio. Ambos términos se usan en nuestro país como sinónimos.

La primera (SI) se refiere al empleo de toda la tecnología posible, dentro de un margen económico aceptable, para establecer las plantaciones y elevar la productividad. Las plantas son de alta calidad genética, las preparaciones de sitio y suelo se han intensificado junto con el control químico de malezas. Se aplican fertilizaciones correctivas, especialmente con boro en las plantaciones de Pino radiata y una mezcla variable de NPKB para Eucalyptus.

En la silvicultura intensiva, la programación del establecimiento de las plantaciones se realiza bajo criterios similares, sin diferenciar en la calidad de los sitios ni de los suelos. Las prescripciones son generales, especialmente el espaciamiento, la preparación de suelo y del sitio y el control de malezas.

En cambio, la silvicultura específica al sitio (SES) se diferencia de la anterior porque para cada sitio o grupo homogéneo de ellos se prescriben regímenes específicos, en forma previa al establecimiento de las plantaciones, que consideran las limitaciones existentes en cada uno de ellos.

En este tipo de silvicultura el análisis de riesgos es un tema importante que sirve para prevenir o aminorar las pérdidas de productividad que pueden experimentar las plantaciones y mejorar así la rentabilidad.

Entre los riesgos más frecuentes que pueden afectar el desarrollo de las plantaciones, pueden mencionarse las sequías o los excesos de agua, causados por las corrientes del Niño y la Niña; la aparición de plagas y enfermedades, los temporales de viento, las temperaturas extremas.

Uno de los principales obstáculos que debe resolver la SES, al planificar los regímenes silvícolas, es proponer métodos que minimicen la influencia de las resistencias ambientales (figura 1), para aumentar así el crecimiento y productividad de las plantaciones, modificando la capacidad de carga del sitio y elevando la curva de crecimiento (12).

Estas resistencias ambientales se encuentran presentes en los diversos componentes de cada ecosistema, sea en el suelo, en la atmósfera más próxima a las plantas, en el clima local, en la topografía, elementos que interactúan y controlan la productividad actual de las plantaciones (13).

La curva actual de crecimiento puede ser modificada, aplicando técnicas silvícolas que reduzcan las restricciones nutricionales e hídricas que se encuentran en el suelo o, bien, modificando algunas propiedades del suelo que faciliten el trabajo del sistema radicular. 
BOSQUE 25(2): 101-113, 2004

Alternativas silvícolas para aumentar la rentabilidad de las plantaciones forestales

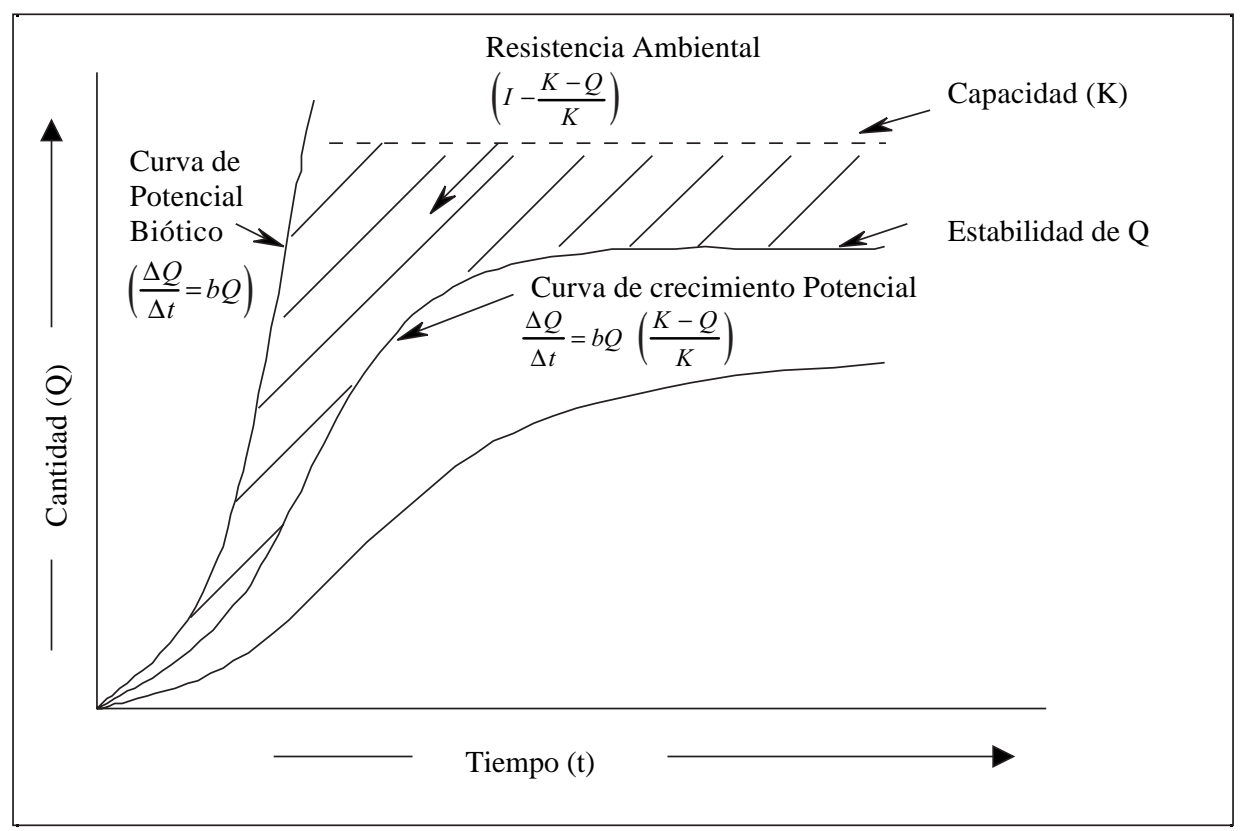

Figura 1. Curvas de crecimiento actual y potencial relacionadas con las resistencias ambientales. Actual and potential growth curves related to the environmental constraints.

Cada sitio presenta una combinación única de resistencias ambientales que restringen su productividad actual, por lo tanto, las prescripciones que se propongan deben incluir los tratamientos y métodos apropiados para controlar y manejar las resistencias ambientales existentes $(9,14)$. La agricultura de precisión tiene un enfoque parecido (15).

Es importante señalar, además, que la calidad del sitio no presenta un valor fijo, más bien fluctúa ampliamente sobre una línea base de productividad (16) y, por lo tanto, el aumento o reducción de la productividad dependerá de la efectividad de los tratamientos que se apliquen en cada sitio.

Los silvicultores tienen, junto con otros especialistas, la oportunidad de aplicar una amplia gama de recursos tecnológicos para reducir las limitaciones del sitio y promover un significativo aumento de la productividad de las plantaciones (17).

El SES permite desarrollar regímenes silvícolas que aumenten la productividad de las plantaciones desde el momento del establecimiento, siendo al mismo tiempo más eficiente en la estructura de costos, lo que se reflejará en la rentabilidad de las plantaciones.

El SES se representa por medio de un ciclo silvícola, en donde los aumentos de productividad se acumulan paso a paso, a lo largo de dicho ciclo, permitiendo elevar la productividad mediante respuestas tipo I y II (18). Otros autores han perfeccionado más estos estudios y han generado otras curvas adicionales $(19,20)$.

Las curvas del tipo I y II son respuestas de diferente magnitud, frente a la aplicación de diversos tratamientos culturales, que permiten aumentar significativamente la productividad de las plantaciones. Los tratamientos aplicados dependerán de las resistencias ambientales que existan en un sitio determinado.

En la curva del tipo I la respuesta inicial a un tratamiento (control de malezas en este caso) es significativamente superior al control, pero al cabo de un tiempo los incrementos corrientes anuales son similares y las curvas son paralelas, manteniéndose la ganancia obtenida a lo largo de la rotación.

En cambio, en la curva del tipo II, los tratamientos aplicados promueven incrementos corrientes anuales que son significativamente mayores al testigo, generando curvas divergentes que pueden duplicar o triplicar la productividad. Sin embargo, entre ambos tratamientos, las respuestas son del tipo I. 
BOSQUE 25(2): 101-113, 2004

Alternativas silvícolas para aumentar la rentabilidad de las plantaciones forestales

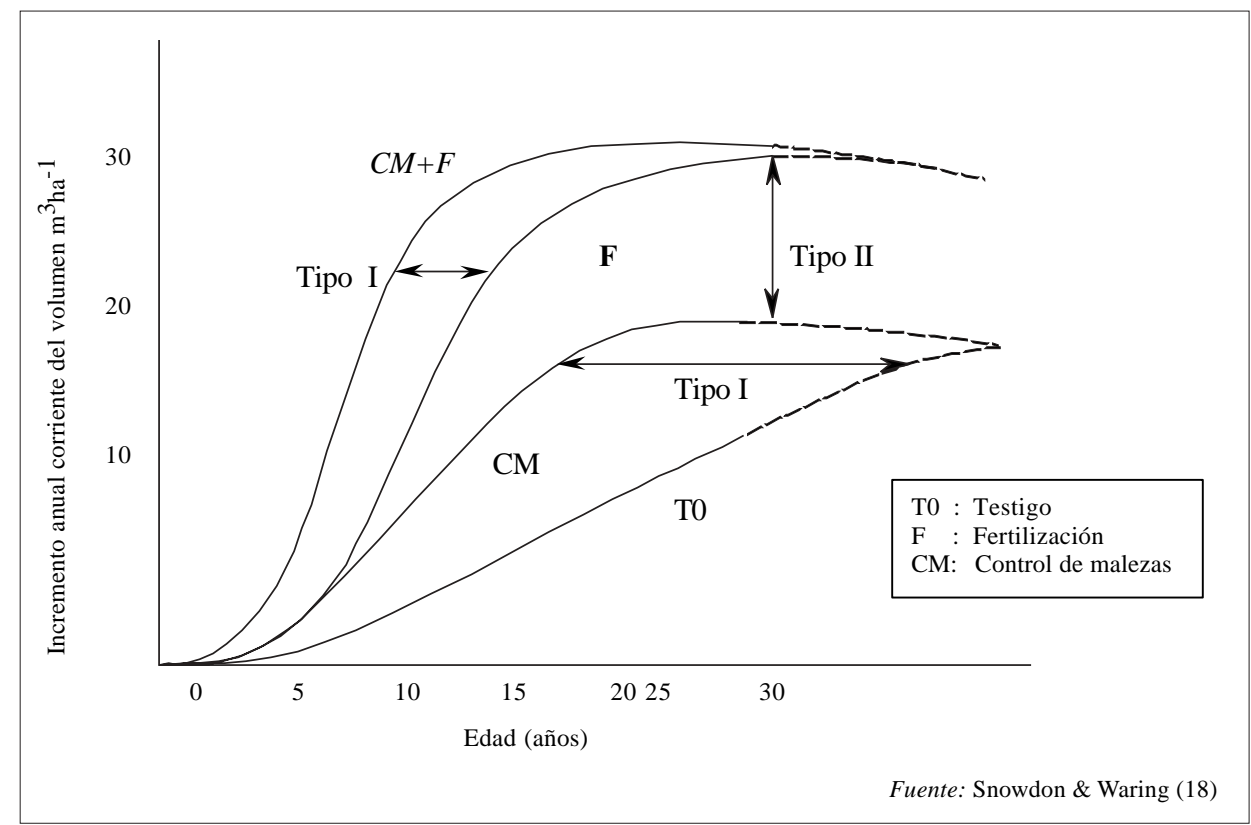

Figura 2. Curvas de Tipo I y Tipo II.

Type I and II growth curves.

Elementos necesarios para elaborar una estrategia silvicultural específica al sitio. Entre los elementos necesarios para elaborar una estrategia que contribuya a elevar la rentabilidad de las plantaciones, se encuentra la información física y química de suelos, que contribuye a identificar sus principales limitaciones.

Esta información, debidamente interpretada, es fundamental para preparar las prescripciones que conforman los regímenes silvícolas, especialmente en la fase de establecimiento. Guiará en la selección de la maquinaria apropiada para preparar el sitio y el suelo; para ayudar a los viveristas a la selección de las plantas-tipo adaptadas a sitios específicos, como también afinar la selección del material genético que se plantará. Comienza, desde este punto, a generarse una ganancia, porque los errores técnicos, que pueden producirse al establecimiento, son minimizados.

Información básica de suelo. La información necesaria para desarrollar un SES requiere identificar, en detalle, los factores que controlan la aireación del suelo, la disponibilidad de agua y nutrientes y el volumen de suelo disponible para el arraigamiento de las plantas (16).
Los suelos de nuestro país, en especial entre la V y X Región, se caracterizan por presentar una gran variabilidad, en sus características físicas, químicas y biológicas. El sobreuso ha generado una elevada tasa de erosión principalmente hídrica. Estos procesos erosivos eliminaron el material fino, principalmente arcillas, limos y materia orgánica, los cuales son muy importantes para mantener una alta productividad del suelo.

Por otra parte, la cordillera de la costa, el valle central y la precordillera andina inducen cambios climáticos fuertes, generando una gran cantidad de distritos agroclimáticos en el territorio (21).

Los materiales geológicos que se encuentran en las distintas regiones son de variada composición química, influyendo significativamente en las actuales características físicas y químicas de los suelos. El efecto a lo largo del tiempo, del clima y de la vegetación sobre la formación de los horizontes de suelos, ha desaparecido debido a la erosión (22).

Como resultado de ello, el contenido de materia orgánica es muy bajo en la mayoría de los suelos entre la V y IX Región. Lo mismo ocurre con la densidad aparente del suelo, que suele ser superior a 1,5 ton $/ \mathrm{m}^{3}$ (cuadro 1 ). 
BOSQUE 25(2): 101-113, 2004

Alternativas silvícolas para aumentar la rentabilidad de las plantaciones forestales

\section{CUADRO 1}

Características de suelo que influyen en el desarrollo de las plantaciones.

Soil characteristics influencing plantation growth.

\begin{tabular}{|c|c|c|c|c|c|c|}
\hline Tipo de Suelo & $\begin{array}{c}\text { Contenido } \\
\text { de M. O. } \\
(\%)\end{array}$ & $\begin{array}{c}\text { Espesor } \\
\text { de suelo } \\
(\mathrm{cm})\end{array}$ & $\begin{array}{l}\text { Densidad } \\
\text { aparente } \\
\left(\text { ton } / \mathrm{m}^{3}\right)\end{array}$ & $\begin{array}{l}\text { Precipitación } \\
\text { promedio } \\
(\mathrm{mm})\end{array}$ & $\begin{array}{c}\text { Agua } \\
\text { Aprovechable } \\
(\%)\end{array}$ & $\begin{array}{l}\text { Drenaje } \\
\text { interno }\end{array}$ \\
\hline Graníticos & $0,8-6,4$ & $30-200$ & $1,3-1,8$ & $310-1.600$ & $10-30$ & bueno \\
\hline Metamórficos & $0,9-7,0$ & $25-150$ & $1,4-1,6$ & $420-1.800$ & $14-28$ & lento \\
\hline Arenas volcánicas & $0,4-4,2$ & $25-70$ & $1,6-1,9$ & $350-1.200$ & $5-10$ & rápido \\
\hline Cenizas volcánicas nuevas & $8,0-17,9$ & $50-280$ & $0,6-1,1$ & $900-1.800$ & $30-50$ & rápido \\
\hline Cenizas volcánicas antiguas & $3,7-6,7$ & $30-70$ & $0,9-1,2$ & $1.100-1.600$ & $20-40$ & lento \\
\hline Terrazas marinas & $2,5-4,8$ & $45-90$ & $1,2-1,8$ & $1.100-1.300$ & $15-30$ & muy lento \\
\hline
\end{tabular}

Toro, Enríquez, Mora, 2003. Informe en preparación.

Los procesos erosivos que afectan a muchos suelos de la costa son responsables de la baja tasa de infiltración que presentan, almacenando una cantidad de agua inferior a los suelos originales.

El espesor útil de los suelos, en las cenizas volcánicas antiguas, no supera los 30 a $40 \mathrm{~cm}$ de profundidad. Bajo este suelo, se encuentra una capa potente de brecha o conglomerado volcánico que actúa como una loza, o un grueso harpan. En otros casos, como ocurre en los suelos graníticos y las arenas volcánicas, el material que se encuentra bajo los 30 a $40 \mathrm{~cm}$ de profundidad, está fuertemente meteorizado (maicillo), pero tiene contenidos muy bajos de materia orgánica y nitrógeno, afectando el crecimiento de las plantas.

El bajo contenido de materia orgánica, la elevada densidad aparente, los problemas de drenaje, la baja capacidad de almacenamiento de agua, que se encuentra en muchos suelos, constituyen resistencias ambientales que influyen en las actuales curvas de crecimiento de las plantaciones forestales.

La formación de una base de datos de suelo adquiere mucha importancia, junto con el desarrollo de mapas y planos digitales, con sus respectivas leyendas interpretativas, que no sólo indiquen las características físicas, químicas de las limitaciones más relevantes del suelo, sino que deben interpretarse para las prescripciones de preparación de sitios y suelo y los métodos apropiados para establecer las plantaciones.
Primeros pasos para aumentar la productividad de las plantaciones, creación de la planta tipo y selección del material genético adaptado al sitio. Los sectores que se plantan cada temporada presentan grandes diferencias en cuanto a suelo, clima y topografía, como se desprende del cuadro 1. Las plantas deben ser acondicionadas en forma óptima en los viveros para establecerlas en sitios cuyas limitaciones están definidas (23). Al producir plantas de calidad y adaptadas al sitio, el comportamiento de las plantaciones mejorará significativamente (24).

La información específica de los sitios y suelos que serán plantados (regímenes de temperatura, humedad, viento, que predominan, características nutricionales y físicas del suelo, etc.) permite a los viveristas acondicionar las plantas para entregar plántulas con diámetros de cuello, altura, volumen radicular, contenidos nutricionales, definidos previamente al evaluar las condiciones de terreno.

Al mismo tiempo, el material genético debe estar plenamente identificado y conocer el comportamiento en los distintos tipos de suelo.

Segundo paso para concretar el aumento de productividad: manejo inicial de las plantaciones. La información de suelo es de gran importancia para concretar el potencial de crecimiento de las plantas e iniciar un proceso de desarrollo y crecimiento que se reflejará en un aumento de la productividad de las plantaciones (10). 
BOSQUE 25(2): 101-113, 2004

Alternativas silvícolas para aumentar la rentabilidad de las plantaciones forestales

Dicha información permite seleccionar opciones para preparar el sitio y preparar el suelo. Las alternativas pueden fluctuar entre distribuir los desechos que quedan sobre la superficie después de cosechar, limpiar el terreno (dependiendo si es forestación o reforestación), o bien quemarlos.

La opción de fajear los residuos siguiendo las curvas de nivel o en forma perpendicular a ellas, distribuirlos homogéneamente para cubrir toda la superficie, o quemarlos, afectará el crecimiento inicial de las plantaciones, porque influirá en las tasas de infiltración de agua, en las tasas de erosión, etc.

La cantidad de agua que se almacene, según las características físicas del suelo, influirá en la tasa de entrega de agua a la planta. El tiempo que permanezca disponible el agua dentro del suelo influirá en los diversos procesos fisiológicos de la planta, como lo son la fotosíntesis, la respiración y la producción de carbohidratos para formar compuestos orgánicos más complejos. También contribuye a formar compuestos secundarios que le sirven de defensa para protegerse del ataque de agentes patógenos (25).

El contenido de agua en el suelo influye en el régimen de temperatura de este, y por lo tanto, influirá en el inicio y duración estacional de la actividad radicular especialmente en los procesos de absorción de nutrientes y agua (26).

Algunos ejemplos. Muchos sectores ubicados en el sector costero de la Octava Región presentan una excesiva humedad, producto del drenaje interno que es muy lento por la presencia de texturas arcillosas densas. Cuando el manejo de residuos cubre todo el suelo, en enero se han registrado temperaturas que fluctúan entre 8 a $10^{\circ} \mathrm{C}$ a $10 \mathrm{~cm}$ de pro- fundidad. Esto afecta significativamente la actividad de las raíces en sus procesos de absorción y disminuye por lo tanto el crecimiento de las plántulas.

Una situación opuesta ocurre en sectores ocupados por arenas volcánicas, con los residuos dispuestos en fajas. Aquí la temperatura del suelo es superior a $50^{\circ} \mathrm{C}$ en verano y afecta seriamente el trabajo del sistema radicular y la cantidad de agua disponible en el suelo. De hecho, el crecimiento se detiene a partir del mes de enero y se reanuda en abril. Al modificar el manejo de residuos cubriendo toda la superficie se logra una mejor protección al suelo, mantiene una mayor humedad cerca de la superficie, lo que permite además disminuir la temperatura de éste.

Tercer paso para aumentar la productividad de las plantaciones. El control químico de malezas es un eficaz medio para redistribuir el agua y los nutrientes que se encuentran en el suelo y dirigirlo hacia las especies de interés. Puede realizarse en fajas, en tazas o en toda la superficie. Esta aplicación se efectúa antes de la plantación y una o dos temporadas después de establecida.

Los resultados en cuanto a crecimiento son significativos, alcanzando a duplicar el crecimiento de aquellas plantas en donde las malezas no son controladas o este control se ha hecho en forma tardía $(27,28)$.

En un estudio efectuado en Valdivia, en una plantación de pino radiata, que crecía en suelos de origen volcánico, se detectó que las malezas competían seriamente por nutrientes y la producción de biomasa por ha mostraba una fuerte competencia por los nutrientes (cuadro 2).

\section{CUADRO 2}

Producción de biomasa aérea y uso de nutrientes entre dos plantaciones de $P$. radiata de cinco años de edad, incluyendo la vegetación acompañante.

Above-ground biomass production and nutrient use between two radiata pine plantations five years old, including weeds growing at the same site.

\begin{tabular}{|l|c|c|c|c|c|}
\hline \multirow{2}{*}{ Especies } & Biomasa aérea & Nitrógeno & Fósforo & Potasio & \multirow{2}{*}{ Boro } \\
\cline { 2 - 6 } & (ton/ha) & \multicolumn{4}{|c|}{ (kg/ha) } \\
\hline P. radiata, sitio A & 7,71 & 64,8 & 7,6 & 32,3 & 0,07 \\
Matorral & 5,48 & 49,7 & 5,8 & 54,3 & 0,10 \\
P. radiata, sitio B & 8,82 & 76,8 & 7,0 & 29,8 & 0,13 \\
Pastos & 3,81 & 74,3 & 8,9 & 49,5 & 0,08 \\
\hline
\end{tabular}

Fuente: Mora y Toro (27). 
BOSQUE 25(2): 101-113, 2004

Alternativas silvícolas para aumentar la rentabilidad de las plantaciones forestales

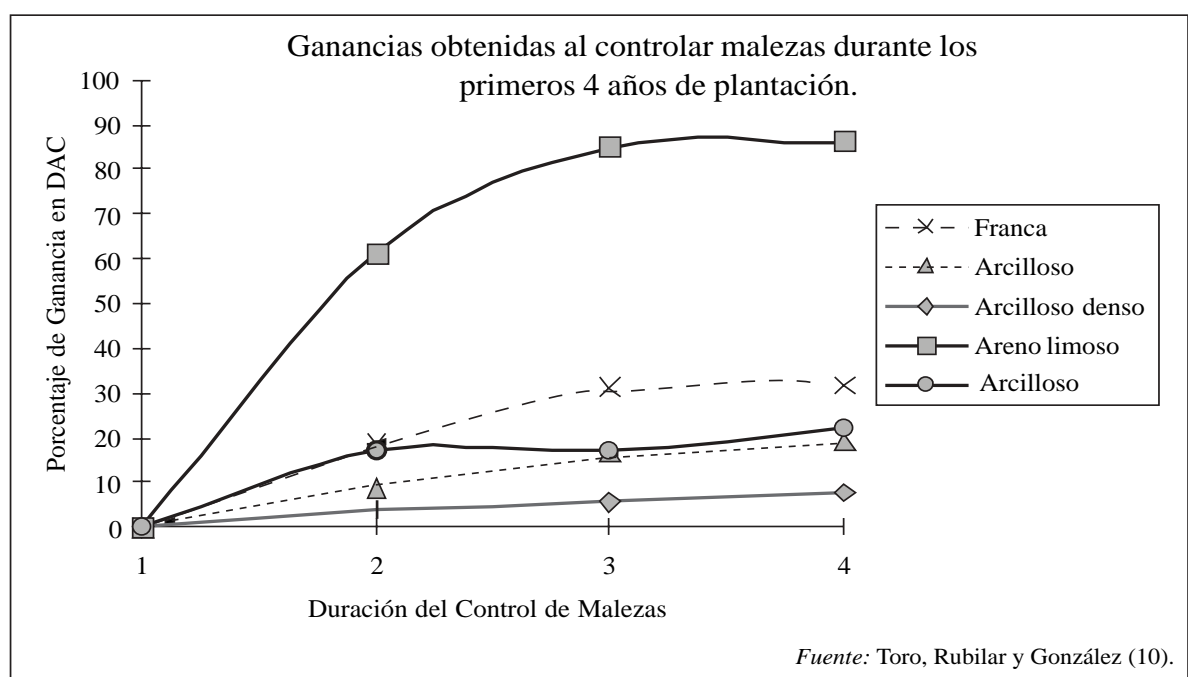

Figura 3. Ganancias en DAC obtenidas al controlar malezas durante cuatro años seguidos, en suelos con diferentes texturas.

Gains in DAC (diameter at collar height) obtained after herbicide applications for a four-year period in contrastingly textured soils.

La cantidad de materia seca producida por las malezas constituye una parte importante de la producción de biomasa en ambos sitios. También la cantidad de nutrientes que almacenan es elevada. Desde un punto de vista ecológico, el rol de esta vegetación es importante porque evitan que los nutrientes desaparezcan del sitio. Sin embargo, para promover una mayor productividad y rentabilidad de las plantaciones, el desarrollo de la vegetación acompañante debe controlarse en un grado tal, que una fracción importante de los nutrientes y producción de biomasa sea canalizada hacia las plantaciones.

Entre las conclusiones que entregó este estudio, destaca la fuerte competencia que realizan las malezas, tanto el pasto como los matorrales, con las plantaciones de pino radiata. Sobresale el hecho que la extracción y acumulación de nutrientes por parte de las malezas es significativo.

Existe una excelente oportunidad para mejorar la productividad de las plantaciones por intermedio del control químico. Sin embargo, las respuestas que se logren estarán en directa relación con las características de cada sitio (figura 3 ).

La respuesta al control de malezas depende en gran medida de la textura del suelo. Se ha encontrado que las texturas areno-limosas y francas favorecen una buena respuesta al control químico de las malezas, exhibiendo las plantas una respuesta favorable en el diámetro. En cambio, las texturas arcillosas, en especial aquellas muy densas, influyen negativamente en el crecimiento en diámetro de las plantaciones de pino radiata.

Fertilización y control de malezas. La fertilización en Chile se aplica en las plantaciones de pino radiata para corregir deficiencias ya existentes en el suelo. Sólo después de transformarse en una práctica rutinaria la aplicación de herbicidas químicos, en la temporada de 1994, el efecto de la aplicación de fertilizantes en conjunto con el control de malezas, empezó a mostrar respuestas significativas.

Tolenaar (29) fue el primero en controlar las deficiencias de boro presentes en las plantaciones chilenas de $P$. radiata, ubicadas en el sector de Linares, en la Séptima Región.

La aplicación de cobre en plantaciones de pino radiata entre 3 y 5 años de edad promueve una significativa respuesta en altura, en predios ubicados en la zona de Florida y Santa Juana, ambos en la Octava Región ${ }^{1}$.

1 Carlos Yáñez, y Edith Fredes. Forestal Bío-Bío, comunicación personal, 2003. 
BOSQUE 25(2): 101-113, 2004

Alternativas silvícolas para aumentar la rentabilidad de las plantaciones forestales

Respuestas a la aplicación de nitrógeno, fósforo, boro y cobre, en plantaciones entre 1 y 6 años de edad, en la zona de Quirihue, son muy significativas en altura y diámetro ${ }^{2}$. Otros sectores, como la costa de Arauco, responden mejor al control de malezas no así a la fertilización ${ }^{3}$. En cambio, las plantaciones de E. globulus y E. nitens reciben una fertilización al establecimiento que considera una mezcla de NPKB, cuya cantidad varía según el tipo de suelos (10).

Curvas de respuesta tipos I y II. Curvas de respuesta del tipo II, similares a las propuestas por Snowdon y Waring (18), se encontraron en suelos de diversas texturas en la Octava Región (figura $4 \mathrm{a}$, figura $4 \mathrm{~b}$, figura $4 \mathrm{c}$.). El control de malezas durante los primeros tres años de crecimiento marca una importante diferencia en las tasas de crecimiento, especialmente a partir del tercer año. En cenizas volcánicas recientes, la mejor respuesta se obtuvo controlando las malezas durante los tres primeros años. Este tratamiento promovió respuestas del tipo II. Al controlar las malezas durante la primera temporada se obtuvo una respuesta del tipo I. De acuerdo a estos resultados, el tipo de respuesta que se obtenga dependerá de la intensidad y duración del control químico.

En suelos arcillosos se obtuvo una respuesta similar. Para ello es necesario aplicar herbicidas durante tres años sucesivos, controlando toda la superficie o también en faja continua y ancho variable.

En los suelos arcillosos, se comparó el efecto de distintas formas de control, en tazas, en fajas y superficie total. Respuestas del tipo II se obtuvieron controlando las malezas en toda la superficie.

Por otra parte, al ser controladas las malezas durante las dos primeras temporadas de crecimiento en suelos de textura arenosa, se observaron también respuestas muy significativas del tipo II.

Cuarto paso, inicio del manejo intermedio de las plantaciones. Hasta ahora el manejo inicial se ha concentrado en acelerar el crecimiento de las plantaciones, para cerrar las copas y ocupar más rápido el sitio. El espaciamiento inicial y los raleos

2 Mauricio Peña. Forestal Millalemu, comunicación personal, 2003.

3 José Manuel Rebolledo, Bosques Arauco, comunicación personal, 2003. que vienen a continuación (raleos a desecho y comerciales) tienen como objetivo central redistribuir los recursos hídricos y nutricionales a los árboles que permanecerán en pie hasta la cosecha final (30). De este modo, el aumento en volumen y el mejoramiento en calidad de la madera se logra con regímenes silvícolas que consideren las características específicas de los sitios y los objetivos de producción (31). Las podas mejoran la calidad de la madera que se ubica en el segmento libre de ramas.

Una forma de mantener las ganancias en crecimiento logradas hasta el cierre de copas, e incluso elevarlas, consiste en ralear antes de que empiece la competencia interespecífica y evitar así una reducción en las tasas de crecimiento en diámetro (DAP), en el área basal individual y finalmente en el volumen individual de los árboles.

La figura 5 muestra la evolución en el diámetro de las ramas más gruesas en plantaciones de $P$. radiata, según las características de cada sitio.

Se observa que el diámetro de la rama más gruesa, que controla el crecimiento del cilindro defectuoso, varía entre plantaciones que tienen la misma edad e igual densidad inicial y que crecen en sitios de distinta calidad.

Plantaciones establecidas en sitios diferentes, a los cinco años de edad, muestran diferencias en diámetro de rama que alcanzan a $1 \mathrm{~cm}$. Si la primera poda se inicia tomando como variables la edad, altura y el diámetro (DAP) y no se considera el diámetro de las ramas más gruesas, puede promoverse una pérdida en la calidad de la madera.

La severidad de la poda puede provocar diversas respuestas en el crecimiento de los árboles. Se han encontrado, en la VIII Región, diferentes respuestas; por ejemplo, un aumento del crecimiento en DAP, en otras áreas una disminución severa o bien una tasa de crecimiento similar a la que tienen los árboles no podados.

La estructura de la copa, especialmente la cantidad de follaje, el índice de área foliar, la longevidad de las acículas, la eficiencia fotosintética del follaje, reacciona en forma diferente, según sea la calidad del sitio (13). Por lo tanto, una poda realizada a destiempo se convierte en un gasto innecesario, disminuye el crecimiento del bosque y afecta la calidad de la madera.

Las empresas, de acuerdo a sus objetivos de producción, realizan diversos tipos de raleo, para 


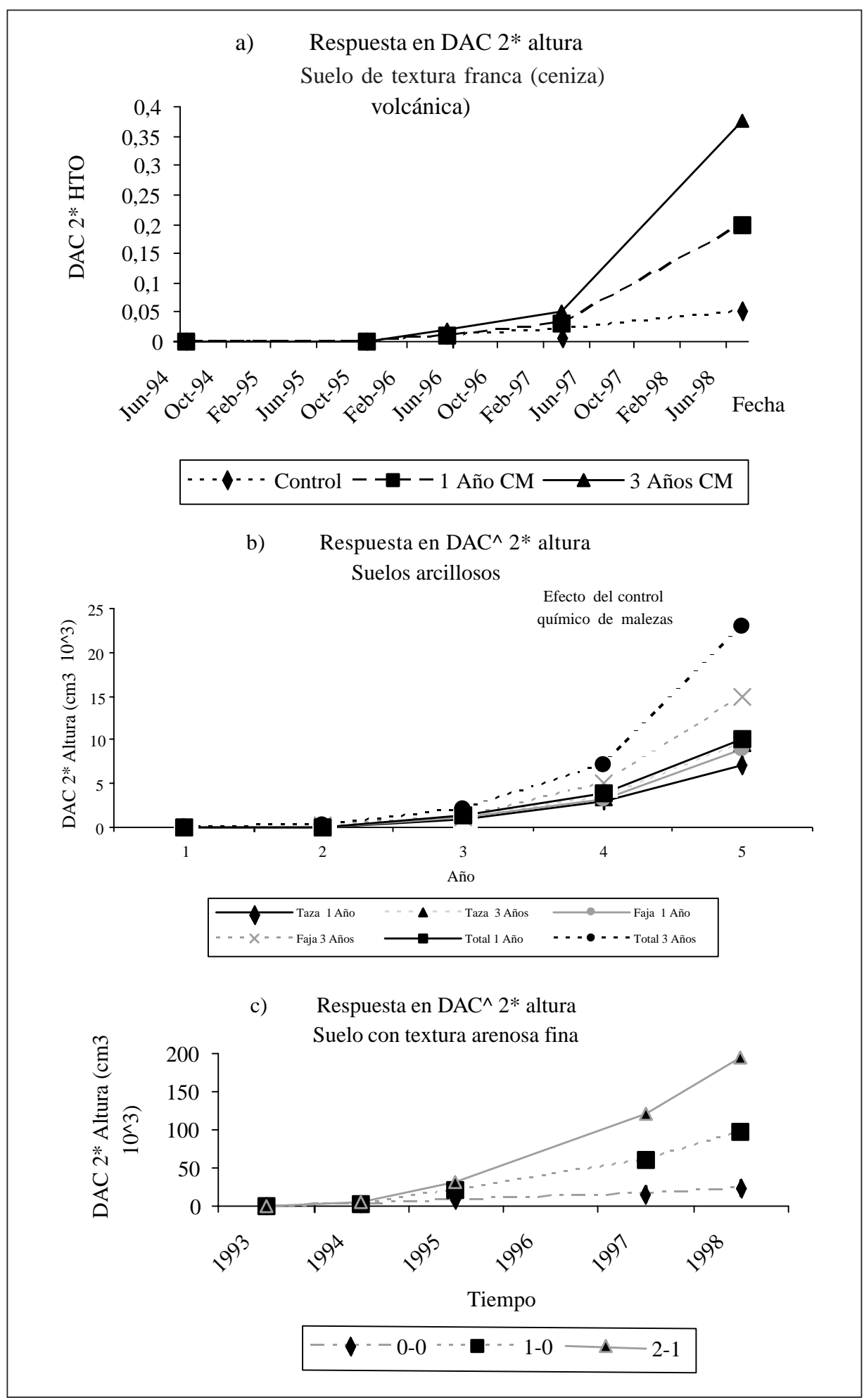

Figura 4. Respuestas tipo I y tipo II en suelos de textura a) franca de cenizas volcánicas, b) arcillosa y c) arenosa fina.

Type I and II responses from: a) loamy volcanic ash, b) clay soils, c) fine sandy soils. 


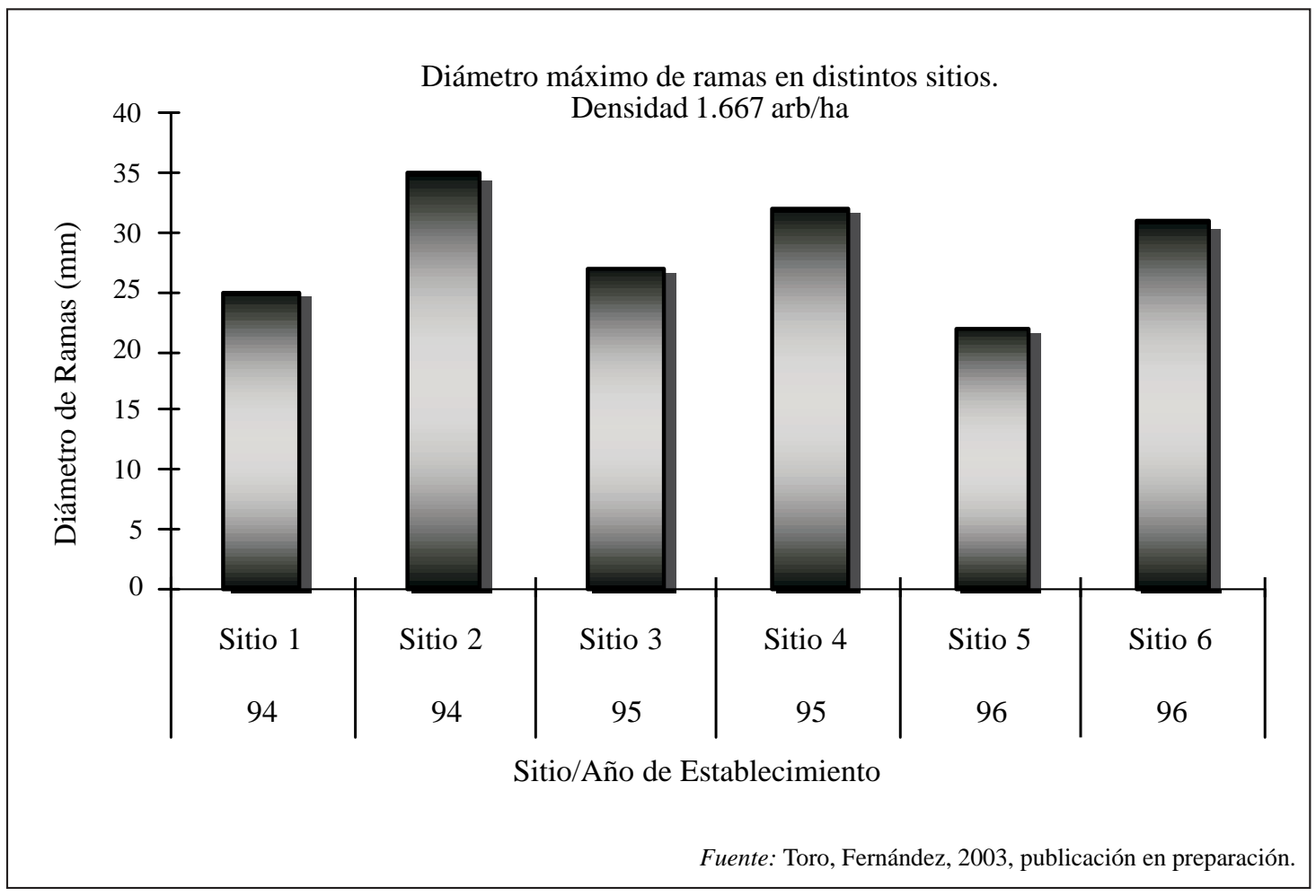

Figura 5. Evolución del diámetro de las ramas más gruesas, con relación a la edad y al sitio.

Branch diameter evolution in relation with age and sites.

alimentar sus plantas de celulosa. Otras buscan producir madera aserrable, libre o no de nudos y la generación de diversos productos. Existen muy pocos estudios en el país acerca de los efectos producidos por raleos combinados con una fertilización.

Raleos tempranos combinados con fertilización. Un estudio efectuado en el sector de Santa Juana (Octava Región) comparó el efecto de dejar distintas densidades (700, 800 y 850 árboles/ha) raleando un sector a los 5 años y otro a los 9 años de edad (figura 6).

El resultado obtenido transcurridos 5 años indicó que para ese sitio era preferible ralear y fertilizar a los 5 años, dejando en pie 800 árboles, en el primer raleo a desecho. La ganancia en volumen individual y total fue de $71 \%$ y $80 \%$, respectivamente. Al dejar en pie 850 árb/ha, la ganancia obtenida fue de $64 \%$ y $45 \%$ con respecto a la plantación raleada a los 9 años de edad y que además no fue fertilizada. En este, sitio al propor- cionarle más recursos hídricos y nutricionales a través del control de malezas y fertilización, se logran resultados que permiten extraer entre 100 a 150 árboles adicionales con superior volumen individual y total.

Procesos ecofisiológicos. Los procesos ecofisiológicos que se desarrollan en las plantaciones necesitan estudiarse en detalle, para cuantificar y evaluar el efecto que producen los distintos tratamientos (25). Al conocer los procesos fisiológicos que se producen en el interior de las plantas, es posible identificar aquellas actividades silvícolas que generan los cambios deseados y conducir las actividades silviculturales hacia un objetivo conocido, evaluado, que favorecerá el aumento de productividad. Por otra parte, al conocer las consecuencias que tiene cada tratamiento, o conjunto de ellos, es posible tomar los resguardos apropiados y evitar hacer tratamientos que signifiquen un costo elevado y no producen los retornos esperados. Esto conduce también a un aumento de la rentabilidad. 
BOSQUE 25(2): 101-113, 2004

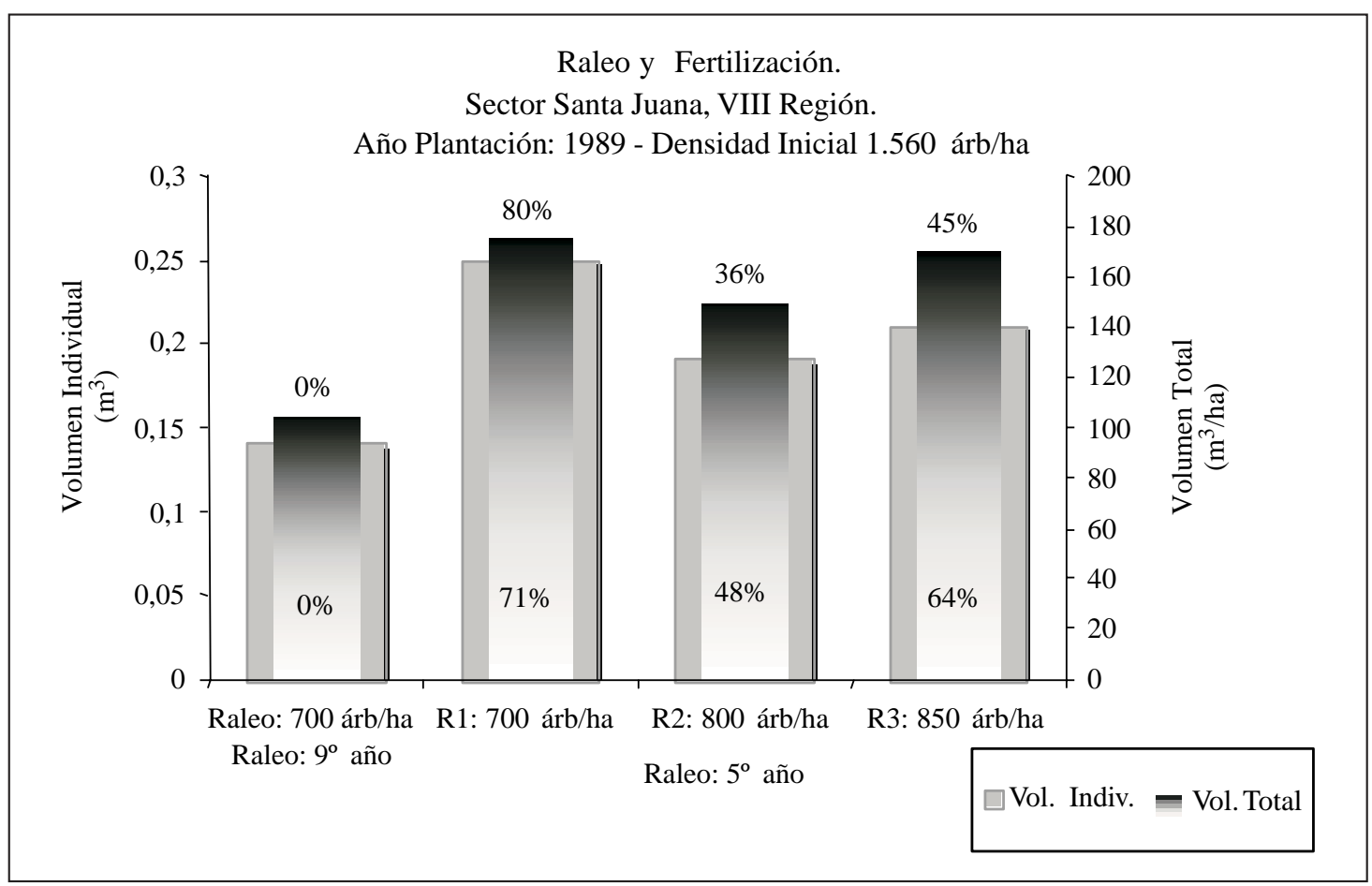

Figura 6. Respuesta en volumen individual y total frente a una combinación de raleo y fertilización aplicada en dos oportunidades y modificando las densidades, en el mismo sitio.

Volume response to thinning and fertilization obtained from different ages and densities of trees at the same site.

El control de malezas, la fertilización, el espaciamiento, el raleo, las podas, la preparación del sitio y el suelo, modifican, aumentando o redistribuyendo la cantidad de recursos que se encuentran en el suelo y que se movilizan internamente dentro de la vegetación, aumentando la disponibilidad de agua, nutrientes y de espacio disponible para evitar la competencia inter e intraespecífica $(19,32)$. La arquitectura de la copa también es modificada, específicamente el área foliar.

La radiación fotosintéticamente activa (RFA) puede ser capturada más eficientemente por el follaje, y al disponer de agua y nutrientes suficientes durante un período más prolongado, la productividad de las plantaciones aumenta (13).

\section{CONCLUSIONES}

Para elevar la productividad de las plantaciones, se requiere desarrollar un ciclo silvícola, que se inicie antes del establecimiento de las plantaciones y finalice después de cada cosecha. Una serie de pasos deben ser seguidos para conocer anticipadamente las limitaciones existentes en los distintos sitios.

Un mapeamiento detallado de suelos, que estratifique el área en estudio en unidades homogéneas, es fundamental desarrollar antes de hacer cualquier planificación. El SIG es de gran utilidad para preparar la información y hacerla accesible a todos los usuarios. La información climática también es importante conocer y evaluar, para que junto con los datos de suelo, se facilite la identificación de las resistencias ambientales del sitio y poder incluirlas en los mapas.

La preparación de regímenes silvícolas, con prescripciones detalladas para cada sector, y el empleo de plantas-tipos, apropiadas para los diferentes sitios, es requisito básico para promover un aumento de la productividad desde el establecimiento.

La silvicultura específica al sitio está orientada a desarrollarse en sectores con características es- 
peciales, aplicando técnicas silvícolas que disminuyan al máximo las resistencias ambientales, proporcionando o redistribuyendo los recursos necesarios sea de agua, nutrientes y radiación, para que las plantaciones aumenten su productividad y sean al mismo tiempo eficientes en costos, lo que influye en la rentabilidad final. Esto se puede lograr con cierta facilidad, si existe la capacidad para identificar los factores que limitan el crecimiento y generar los tratamientos en forma oportuna y sincronizada.

Los estudios ecofisiológicos tienen una gran importancia en el monitoreo de los distintos tratamientos aplicados a las plantaciones. De esta manera, la efectividad de los tratamientos queda rápidamente de manifiesto, pudiendo modificar los regímenes silvícolas si así es requerido.

\section{BIBLIOGRAFIA}

(1) TORO, J., S. P. GESSEL. S. Radiata pine plantations in Chile. In: J. BOYLE and K. KAVANAGEH. Planted Forest Symposium. Contribution to Sustainable Societies, Oregon, USA. New Forest, 1999, vol. 7, N ${ }^{\circ} 1-3$ y vol. 8, $\mathrm{N}^{\circ} 1$.

(2) ALVAREZ, J., J. RODRIGUEZ, D. SUAREZ. 1999. Mejoramiento de la productividad de plantaciones de Pinus radiata $\mathrm{D}$. Don, a través de un método racional de fertilización. Bosque (Chile), 1999, vol. 20, No 1, p. 23-36.

(3) FRANCKE, S. Efecto del manejo de residuos de explotación en el suelo y crecimiento inicial de plantaciones de Pinus radiata, en series de suelo Coreo (Zona Arenales) y Colico (Zona Arauco). Chile Forestal, 1991. Documento Técnico, $\mathrm{N}^{\circ} 52.9 \mathrm{p}$.

(4) GERDING, V., J. SCHLATTER. Estabilidad nutritiva de plantaciones de Pinus radiata D. Don en cinco sitios característicos de la VIII Región. Bosque (Chile), 1999, vol. $20, \mathrm{~N}^{\mathrm{o}} 2$, p. 107-115.

(5) GREZ, R., L. PIEL, R. AÑAZCO. Los análisis químicos en los estudios de suelos forestales. Boletín de la Sociedad del Suelo, No 10, p. 46-64, 1993.

(6) ORTEGA, R. Manejo sitio específico: Aplicabilidad plantaciones forestales. X Silvotecna: Mejoramiento de Productividad de Sitio, Concepción, Chile, 1998. 12 p.

(7) SCHLATTER, J., V. GERDING. Método de clasificación de sitios para la producción forestal, ejemplo en Chile. Bosque (Chile), 1995, vol. 16, No 2, p. 13-20.

(8) SCHLATTER, J., V. GERDING. Productividad en el ejemplo de seis sitios característicos de la VIII Región con Pinus radiata D. Don. Bosque (Chile), 1998, vol. 20, $\mathrm{N}^{\mathrm{o}}$ 1, p. 65-77.

(9) TORO, J. Avances en fertilización en Pino radiata y Eucalyptus en Chile, p. 293-299. Actas Simposio Internacional IUFRO para el Cono Sur. Abril 1995. Universidad Austral de Valdivia, Valdivia.

(10) TORO, J., R. RUBILAR, C. GONZALEZ. Métodos para aumentar la productividad de plantaciones de Pino radiata y Eucalyptus globulus entre las Regiones Séptima y Octava, In: Simposio IUFRO: Manejo Sustentable de los Recursos Forestales, Desafío del siglo XXI. Primer Congreso Latinoamericano. Valdivia, noviembre 1998, 10 p.
(11) TORO, J. Acumulación de calcio en plantaciones de Eucalyptus globulus y E. nitens, p. 244-248. Sociedad Chilena de la Ciencia del Suelo. IX Congreso Nacional de la Ciencia del Suelo. Universidad de Talca, 4-6 de noviembre, 2002, Talca.

(12) BOARDMAN, R. Fast growing species-pattern, process and ageing p. 1-49. In: Symposium on site and productivity of fast growing plantations. IUFRO, Pretoria and Pietermarizburg, South Africa. 1984.

(13) WARING, R.H., S. W. RUNNING. Forest Ecosystems: Analysis at Multiple Scales. Academic Press, San Diego, CA. 1998 , p. 370.

(14) TURNER, J., M. LAMBERT. Site variation in Pinus radiata and implications for site specific management. New Forest, vol. 21, p. 249-282, 2001

(15) ORTEGA, R., L. FLORES. Agricultura de precisión: Introducción al manejo de sitio-específico. Ministerio de Agricultura. Instituto de Investigaciones Agropecuarias, 1999. Centro Regional de Investigación Quilamapu. 146 p.

(16) STONE, E. Site quality and site treatment, p. 4-52. In: Forest Soils \& Treatments Impacts. The University of Tennessee, USA. 1984.

(17) GESSEL, S.P. Site evaluation in forest production and management, pp 326-355. In: SCHLATTER, J. E . Evaluación de la Productividad de los Sitios Forestales. 1981. Actas Reunión de Trabajo, Universidad Austral de Chile. 1981.

(18) SNOWDON, P., H. D. WARING. Long-term nature of growth responses obtained to fertiliser and weed control applied at planting and their consequences for forest management, p. 701-712. In: Symposium on site and productivity of fast growing plantations. IUFRO. Pretoria and Pietermarizburg, South Africa. 1984

(19) ALLEN, H. L., P.M. DOUGHERTY, R.G. CAMPBELL. Manipulation of water and nutrients - practice and opportunity in southern US pine forests. Forest Ecology and Management. 1990, vol. 30, p. 437-328.

(20) RICHARDSON, B. Vegetation management practices in plantation forests of Australia and New Zealand. Canadian Journal Forest Research, 1993, vol. 23, p. 1989-2005.

(21) SANTIBAÑEZ, F., J. URIBE.. Atlas agroclimático de Chile. VI, VII, VII y IX Regiones. Laboratorio de Agroclimatología, Facultad de Ciencias Agrarias y Forestales, Universidad de Chile, CORFO, Santiago, 73 p., 1993.

(22) CARRASCO, P. Suelos forestales de la VIII Región. Chile Forestal, 1989. Documento Técnico. No 41, 4 p.

(23) ROSE, R., W.C. CARLSON. The target seedling concept, p. 1-8. In: Proceeding: Combined meeting western forest nursery associations. U.S.D.A. Forest Service, General Technical Report RM-200, 1990.

(24) ESCOBAR, R. La planta ideal: variables que predicen su comportamiento y factores que lo afectan. 22 p. En: Silvotecna, Forestal Mininco y Fundación Chile. 1994.

(25).WARING, R.H., W.H. SCHLESINGER. Forest Ecosystems: Concepts and Management. Academic Press, Inc. 1985 , p. 340.

(26) SAMPSON, D.A., H.L. ALLEN. Regional influences of soil available water-holding capacity and climate, and leaf area index on simulated loblolly pine productivity. Forest Ecology and Management, 1999, vol. 124, p. 1-12.

(27) MORA, C., J. TORO. Control de Malezas en Plantaciones de $P$. radiata, establecidas en Valdivia, In: $X$ Silvotecna Mejoramiento de la Productividad del Sitio, p. 200-215, junio, 1998, Concepción.

(28) RUBILAR, R. Control de malezas y fertilización de plantaciones de Pinus radiata D. Don establecidas en suelos metamórficos del predio Quivolgo II, Constitución, VII Región. Memoria para optar al título de Ingeniero Fores- 
BOSQUE 25(2): 101-113, 2004

Alternativas silvícolas para aumentar la rentabilidad de las plantaciones forestales

tal, Fac. Cs. Agrarias y Forestales, Universidad de Chile. 1998. $116 \mathrm{p}$

(29) TOLENAAR, H. Deficiencias de boro en plantaciones de pino en la zona central de Chile. Instituto Forestal Latino-Americano de Investigación y Capacitación. Mérida, Venezuela. 1970. Boletín $\mathrm{N}^{\circ}$ 33-34, p. 80-86.

(30) SHEPERD, K. Plantation Silviculture. Martinus Nijhoff Publishers. 1986. 322 p.
(31) NAMBIAR, E.K.S. Relationships between water, nutrients and productivity in Australian forests: Application to wood production and quality. Plant and Soil, 1995, N ${ }^{\circ} 168-169$, p. $427-435$.

(32) NAMBIAR, E.K.S. Interplay between nutrients, water, root growth and productivity in young plantations. Fores Ecology and Management, 1990, vol. 30, p. 213-232.

Recibido: 03.10 .03

Aceptado: 24.06.04 